Received Date : 10-Jul-2016

Revised Date : 30-Aug-2016

Accepted Date : 20 -Sep-2016

Article type : Short Communication

\title{
METABOLIC PROFILE OF INJURED HUMAN SPINAL CORD DETERMINED USING SURFACE MICRODIALYSIS
}

Suliang Chen $\mathrm{PhD}^{1}$, Isaac Phang $\mathrm{MD}^{1}$, Argyro Zoumprouli $\mathrm{MD}^{2}$, * Marios C. Papadopoulos $\mathrm{MD}^{1}$, *Samira Saadoun $\mathrm{PhD}^{1}$

${ }^{1}$ Academic Neurosurgery Unit, St. George's, University of London, U.K. ${ }^{2}$ Neurointensive Care, St. George's Hospital, London, U.K.

*Co-senior authors

Corresponding author. Dr. S. Saadoun, Room 1.234 Jenner Wing, St. George's, University of London, London SW17 0RE, U.K. Phone +44(0)2087254179, Fax +44(0)2087254452

Email.ssaadoun@sgul.ac.uk

Running title. Metabolic profile of injured spinal cord

Keywords. Critical care unit, Human, Kohonen self-organizing maps, Microdialysis, Prognosis, Spinal cord injury

This article has been accepted for publication and undergone full peer review but has not been through the copyediting, typesetting, pagination and proofreading process, which may lead to differences between this version and the Version of Record. Please cite this article as doi: $10.1111 /$ jnc. 13854

This article is protected by copyright. All rights reserved. 
Abbreviations. AIS, American spinal injuries association Impairement Scale; ISP, Intra Spinal Pressure; MD, microdialysis; SCPP, Spinal Cord Perfusion Pressure; SOM, self-organizing map; TSCI, traumatic spinal cord injury

\section{ABSTRACT}

The management of patients having traumatic spinal cord injury (TSCl) would benefit from understanding and monitoring of spinal cord metabolic states. We hypothesized that the metabolism of the injured spinal cord could be visualized using Kohonen selforganizing maps. Sixteen patients with acute, severe spinal cord injuries were studied. Starting within 72 hours of the injury, and for up to a week, we monitored the injury site hourly for tissue glucose, lactate, pyruvate, glutamate and glycerol using microdialysis as well as intraspinal pressure and spinal cord perfusion pressure. A hexagonal Kohonen map, which is an unsupervised, self-organizing topology-preserving neural network, was used to analyze 3,366 hours of monitoring data. We first visualized the different spinal cord metabolic states. Our data show that the injured cord assumes one or more of four metabolic states. Based on their metabolite profiles, we termed these states nearnormal, ischemic, hypermetabolic and distal. We then visualized how patients' intraspinal pressure and spinal cord perfusion pressure affect spinal cord metabolism. This revealed that for more than $60 \%$ of the time, spinal cord metabolism is patientspecific; periods of high intraspinal pressure or low perfusion pressure are not associated with specific spinal cord metabolic patterns. Finally, we determined 
relationships between spinal cord metabolism and neurological status. Patients with complete deficits have shorter periods of near-normal spinal cord metabolic states ( $7 \pm 4 \%$ versus $58 \pm 12 \%, P<0.01$, mean \pm standard error) and more variable metabolic responses (metabolism spread in $70 \pm 11$ versus $40 \pm 6$ hexagons, $P<0.05$ ), whereas patients with incomplete neurological deficits have longer and less variable metabolic responses. By visualizing the microdialysis data, Kohonen maps allow us to see these metabolic responses, and may thus aid us in treating patients with acute spinal cord injuries.

\section{INTRODUCTION}

Traumatic spinal cord injury (TSCI) is a devastating condition that primarily affects young men (Cripps et al. 2011). To date, there is no monitoring from the injury site to guide the management of patients with TSCI in the intensive care unit and, consequently, the management is variable (Werndle et al. 2012, Fehlings et al. 2010). In 2014, we described a technique to monitor intraspinal pressure (ISP) and spinal cord perfusion pressure (SCPP) at the injury site (Werndle et al. 2014). Recently, we employed microdialysis (MD) in addition to ISP and SCPP monitoring, to record hourly the levels of glucose, lactate, pyruvate, glycerol and glutamate from the injury sites of patients with acute TSCI (Phang et al. 2016a). Monitoring from the injury site in TSCI patients is safe(Phang et al. 2016a, Phang et al. 2016b).

This article is protected by copyright. All rights reserved. 
We hypothesize that the injury site assumes different metabolic states that correlate with outcome and employ a multivariate technique to analyze the data. In contrast to the univariate methods used in our first MD paper (Phang et al. 2016a), the multivariate method used here considers all metabolic features simultaneously and, consequently, can identify relationships between them. Each set of hourly metabolite measurements is considered as a five-dimensional vector. A Kohonen self-organizing map (SOM) is used to place these five-dimensional vectors in a two-dimensional grid (Kohonen 2013). The Kohonen SOM preserves topological relationships between the five-dimensional vectors as they are mapped onto the two-dimensional grid thus allowing us to visualize clinically important, non-linear relationships between the vectors.

\section{MATERIALS AND METHODS}

Patients: The study was approved by the National Research Ethics Service (No. 10/H0807/23). All patients gave written consent. Inclusion criteria were: 1 . Severe TSCI defined as American spinal cord injuries Impairment Scale (AIS) grade A - C; 2. Age 18 - 70 years; 3. Time from TSCI to surgery $\leq 72$ hours. Exclusion criteria were: 1 . Patient unable to consent; 2 . Other major injuries or co-morbidities; 3 . Penetrating TSCI. Surgery and early management took place at the neurosurgery unit of St. George's Hospital. Recruitment into the study was discussed with all eligible patients and their families on admission, the patient information sheet was given and the consent form was signed. Details are given elsewhere (Phang et al. 2015, Phang et al. 2016a, Phang et al. 2016b, Werndle et al. 2014).

This article is protected by copyright. All rights reserved. 
Monitoring: ISP catheters (Codman Microsensor Transducer®, Depuy-Synthes, U.K.) and MD (CMA61, CMA-Microdialysis, Sweden) probes were placed on the spinal cord surface at the injury site unless otherwise stated, at the end of surgery to realign and fix the fractured spine. The ISP probe was connected to a Codman ICP box linked via a ML221 amplifier to a PowerLab running LabChart v.7.3.5 (AD Instruments, Oxford, UK). Arterial blood pressure was recorded from a radial artery catheter connected to the Philips Intellivue MX800 bedside monitoring system (Philips, Guildford, UK) in turn connected to the PowerLab system. The ISP and arterial blood pressure signals were sampled at $1 \mathrm{kHz}$ for up to seven days. LabChart was used to analyze the signals and compute SCPP, defined as mean arterial pressure minus ISP. We computed SCPP as mean arterial pressure minus ISP. MD monitoring was started postoperatively in the neurointensive care unit. For MD, CNS perfusion fluid (CMAMicrodialysis, Sweden) was perfused at a rate of $0.3 \mu \mathrm{L} / \mathrm{min}$. MD vials were changed hourly and analysed for glucose, lactate, pyruvate, glycerol and glutamate. Details are given elsewhere (Phang et al. 2015, Phang et al. 2016a, Phang et al. 2016b, Werndle et al. 2014).

Data pre-processing: We define a vector as a set of five metabolite measurements (components). A complete vector has no missing components. An incomplete vector has at least one missing component. Spikes are $>3$ standard deviation rises in metabolite concentrations in all five components at a time point. We first filtered the MD data by removing spikes. We then interpolated incomplete vectors using the nearest neighbor approach. Only sequences of up to three consecutive incomplete vectors were interpolated. The resulting MD dataset comprised 3,366 five-dimensional vectors including 2,620 injury site vectors plus 746 vectors from spinal cord below. This corresponds to 16,830 metabolite measurements of which 644 are interpolated.

This article is protected by copyright. All rights reserved. 
Metabolites were normalized to zero mean and unit standard deviation.

Kohonen analysis: We used Matlab SOM toolbox v.2.0beta (www.cis.hut.fi/somtoolbox/). Grid length and width were computed from the ratio between the eigenvalues of the input data. The grid was initialized using random weight-vectors uniformly distributed between the minimum and maximum input values. The Euclidean distance from each input vector to each weight-vector was calculated. The input vector was placed in the hexagon with the closest weight-vector, termed the best matching unit. The connection weights of the best matching unit and its neighboring hexagons were moved towards the input vector using a Gaussian neighborhood function. The process was repeated for all input vectors. The five-dimensional vectors were thus projected onto the two-dimensional map such that similar vectors clustered near each other.

Statistics: In Figure 3, data are shown as mean \pm standard error and two groups were compared using Student's t-test.

\section{RESULTS}

Demographics: We recruited 16 consecutive patients. Most (15/16) were male and most (13/16) were $<50$ years old. $6 / 16$ had cervical and 10/16 thoracolumbar injuries. On admission, 12/16 patients were AIS A and 4/16 B or C. Average follow-up was 32 weeks (range 2 - 89). At follow-up, 9/16 patients were AIS A and 7/16 B or C. MD data were collected for 29 - 324 hours per patient, yielding a total of 3,366 vectors (2,620 from injury site, 746 from spinal cord below). For details see Table 1.

This article is protected by copyright. All rights reserved. 
Metabolic maps: After mapping all 3,366 metabolic vectors in a $24 \times 43$ hexagonal grid, we produced metabolite heat maps by color-coding hexagons based on mean metabolite level (Fig. 1). The top left quadrant (near-normal) has high glucose, low lactate, medium/low pyruvate, low glutamate and low glycerol. The bottom left quadrant (ischemic) has low glucose, high lactate, medium/low pyruvate, high glutamate and high glycerol and includes two high glycerol (cell death) regions. Both forms of cell death are ischemic, but only one has high glutamate. The bottom right quadrant (hypermetabolic) has low glucose, high pyruvate and high lactate, but no glutamate or glycerol. In the top right quadrant, all metabolites have low levels; this profile, termed 'distal', is explained below. Together, our data indicate four major spinal cord metabolic states after injury.

Patient maps: To characterize each patient's injury site metabolic profile, we color-coded hexagons by patient (Fig. 2A). On the left, each hexagon was colored based on the patient with most vectors in that hexagon. On the right, hexagons containing vectors from more than one patient received one color per patient; the colored area is proportional to the number of vectors. The data show that the injured cord may either adopt only one metabolic state (e.g. P13) or flip between states (e.g. P11). Most (60.6 $\pm 3.7 \%$, mean \pm standard error) of each patient's hexagons lack vectors from other patients (Fig. 2B), i.e. metabolic responses are largely patient specific. Consequently, there is no pattern in the spinal cord perfusion pressure (Fig. 2C) and intracranial pressure (Fig. 2D) maps.

This article is protected by copyright. All rights reserved. 
Neurological outcome: To address the hypothesis that the injury site metabolic profile predicts outcome, we colored hexagons based on the AIS grade on admission (Fig. 3A). Unlike the vectors of the 12/16 AIS A patients, the vectors of the 4/16 AIS B or C patients were nearnormal, i.e. clustered in the top left quadrant. Vectors from below the injury clustered in the top right quadrant, which suggests that spinal cord distal to the injury is metabolically abnormal. In Fig. 3B, hexagons were colored according to the AIS grade at follow-up. Compared with the 10/16 AIS A patients, the 6/16 AIS B or C patients had significantly more hexagons in the top left grid quadrant. Therefore, patients presenting with near-normal injury site metabolism have some neurological function below the injury at follow-up even if classed AIS A on admission. We then quantified the variability of the metabolic response of a patient's injury site by counting the number of hexagons that contain vectors from that patient; few hexagons indicate low variability and many hexagons high variability. Fig. 3C shows significantly fewer hexagons per patient for AIS B or C compared with AIS A at follow-up. Therefore, less severely injured spinal cords, which have more preserved tissue homeostasis, have less variable tissue metabolic responses.

\section{DISCUSSION}

Our key finding is that the injured spinal cord adopts different metabolic states. In some patients, the injury site switches between adverse (ischemic) and favorable (near-normal) states. The spinal cord metabolic states after injury are comparable with the states that the injured brain may adopt including ischemic, infarcted, hyperperfused and near-normal (Timofeev et al. 2011, Nelson et al. 2004, Hutchinson et al. 2015) It is interesting to investigate whether therapeutic interventions after TSCI that maintain the near-normal metabolic state throughout, improve outcome.

This article is protected by copyright. All rights reserved. 
We also showed that the injury site metabolic responses are largely patient-specific. This is in agreement with the idea that, after TSCI, the optimum SCPP varies widely between patients (Phang et al. 2015). These observations suggest that patients should be managed individually rather than by applying universal protocols, such as maintaining mean arterial pressure between $85-90 \mathrm{mmHg}$ for a week after TSCI as recommended by the American Association of Neurological Surgeons (Cozzens et al. 2013). The patient-specific nature of the metabolic response of the injured spinal is comparable to the patient-specific metabolic response of the injured brain (Nelson et al. 2004). Factors that may contribute to the individualistic injury site metabolic responses after TSCI include the degree of spinal cord compression, mechanism of injury, extent of microvascular damage and pre-morbid state e.g. genotype.

A near-normal injury site metabolic state on admission was associated with improved outcome and likely indicates milder injury. Reduced variability of a patient's injury site metabolic profile also correlates with improved outcome and likely indicates less severe disruption of tissue homeostasis. In future studies, Kohonen networks may include tissue inflammatory mediators (Bethea \& Dietrich 2002) and growth factors (Awad et al. 2015), in addition to metabolites, to improve prognostication. A larger study is required to definitively determine whether Kohonen SOMs can be used to improve prognostication after TSCI.

We conclude that analyzing MD data with Kohonen SOMs may aid the management of patients with acute, severe TSCI in the intensive care unit.

This article is protected by copyright. All rights reserved. 
Involves human subjects: Yes

If yes: Informed consent \& ethics approval achieved: Yes $\Rightarrow$ if yes, please ensure that the info "Informed consent was achieved for all subjects, and the experiments were approved by the local ethics committee." is included in the Methods.

ARRIVE guidelines have been followed:

Yes

$\Rightarrow$ if No, skip complete sentence

$\Rightarrow$ if Yes, insert "All experiments were conducted in compliance with the ARRIVE guidelines."

Conflicts of interest: None

$\Rightarrow$ if 'none', insert "The authors have no conflict of interest to declare."

$\Rightarrow$ otherwise insert info unless it is already included

\section{ACKNOWLEDGEMENTS}

We thank St. George's Hospital, King's College Hospital and Hurstwood Park Neurological Centre neurosurgical departments helped recruit patients. The anesthetic, neuro-intensive care and operating theatre staff at St. George's Hospital helped with data collection. The study was funded by Wings for Life Spinal Cord Research Foundation, Neurosciences Research Foundation (Fletcher Fund) and the London Deanery.

\section{CONFLICTS OF INTEREST}

None.

\section{REFERENCES}

Awad, B. I., Carmody, M. A. and Steinmetz, M. P. (2015) Potential role of growth factors in the management of spinal cord injury. World Neurosurg, 83, 120-131.

Bethea, J. R. and Dietrich, W. D. (2002) Targeting the host inflammatory response in traumatic

This article is protected by copyright. All rights reserved. 
spinal cord injury. Curr Opin Neurol, 15, 355-360.

Cozzens, J. W., Prall, J. A. and Holly, L. (2013) The 2012 Guidelines for the Management of Acute Cervical Spine and Spinal Cord Injury. Neurosurgery, 72 Suppl 2, 2-3.

Cripps, R. A., Lee, B. B., Wing, P., Weerts, E., Mackay, J. and Brown, D. (2011) A global map for traumatic spinal cord injury epidemiology: towards a living data repository for injury prevention. Spinal Cord, 49, 493-501.

Fehlings, M. G., Rabin, D., Sears, W., Cadotte, D. W. and Aarabi, B. (2010) Current practice in the timing of surgical intervention in spinal cord injury. Spine (Phila Pa 1976), 35, S166173.

Hutchinson, P. J., Jalloh, I., Helmy, A. et al. (2015) Consensus statement from the 2014 International Microdialysis Forum. Intensive Care Med, 41, 1517-1528.

Kohonen, T. (2013) Essentials of the self-organizing map. Neural Netw, 37, 52-65.

Nelson, D. W., Bellander, B. M., Maccallum, R. M., Axelsson, J., Alm, M., Wallin, M., Weitzberg, E. and Rudehill, A. (2004) Cerebral microdialysis of patients with severe traumatic brain injury exhibits highly individualistic patterns as visualized by cluster analysis with self-organizing maps. Crit Care Med, 32, 2428-2436.

Phang, I., Werndle, M. C., Saadoun, S., Varsos, G., Czosnyka, M., Zoumprouli, A. and Papadopoulos, M. C. (2015) Expansion duroplasty improves intraspinal pressure, spinal cord perfusion pressure, and vascular pressure reactivity index in patients with traumatic spinal cord injury: injured spinal cord pressure evaluation study. J Neurotrauma, 32, 865874.

Phang, I., Zoumprouli, A., Papadopoulos, M. C. and Saadoun, S. (2016a) Microdialysis to optimize cord perfusion and drug delivery in spinal cord injury. Ann Neurol, In press.

This article is protected by copyright. All rights reserved. 
Phang, I., Zoumprouli, A., Saadoun, S. and Papadopoulos, M. C. (2016b) Safety profile and probe placement accuracy of intraspinal pressure monitoring for traumatic spinal cord injury: Injured Spinal Cord Pressure Evaluation study. J Neurosurg Spine, EPub.

Timofeev, I., Carpenter, K. L., Nortje, J. et al. (2011) Cerebral extracellular chemistry and outcome following traumatic brain injury: a microdialysis study of 223 patients. Brain, 134, 484-494.

Werndle, M. C., Saadoun, S., Phang, I. et al. (2014) Monitoring of spinal cord perfusion pressure in acute spinal cord injury: initial findings of the injured spinal cord pressure evaluation study. Crit Care Med, 42, 646-655.

Werndle, M. C., Zoumprouli, A., Sedgwick, P. and Papadopoulos, M. C. (2012) Variability in the treatment of acute spinal cord injury in the United Kingdom: results of a national survey. J Neurotrauma, 29, 880-888.

\section{FIGURE LEGENDS}

Fig. 1. Metabolite heat maps. (A) Glucose, (B) Lactate, (C) Pyruvate, (D) Glycerol, and (E) Glutamate, with corresponding color scales below. Color in each hexagon represents the mean of the values in that hexagon.

Fig. 2. Patient maps. (A) Left - Patient color code, Right - each hexagon is colored according to each patient who has contributed vectors with the colored area proportional to the number of vectors. (B) \% hexagons that only have vectors from one patient. (C) Left - Spinal cord perfusion pressure (SCPP) and Right - Intraspinal pressure (ISP) with corresponding color scales. Each hexagon is colored according to its mean value.

This article is protected by copyright. All rights reserved. 
Fig. 3. Neurological outcomes. (A) AIS grade on admission, and (B) AIS grade at follow-up, with corresponding color scales below. 'Distal' is spinal cord distal to the injury site. (C) Rose plots showing number of hexagons in each grid quadrant for different AIS grades at follow-up (mean \pm standard error). Significant difference between AIS A vs. B or $\mathrm{C}$ at $\mathrm{P}<0.05$. (D) Number of hexagons for AIS grade A vs. AIS grade B or C patients. Mean \pm standard error. * $\mathrm{P}$ $<0.05$.

Table 1. Patient details, neurological status and monitoring details.

\begin{tabular}{lllllllll}
\hline Pt. & $\begin{array}{l}\text { Age } \\
\text { no }\end{array}$ & Sex & $\begin{array}{l}\text { Injury } \\
\text { level }\end{array}$ & $\begin{array}{l}\text { Pre-op } \\
\text { AlS }\end{array}$ & $\begin{array}{l}\text { Follow- } \\
\text { up AlS }\end{array}$ & $\begin{array}{l}\text { Follow } \\
\text {-up }(\mathrm{w})\end{array}$ & $\begin{array}{l}\text { Injury to } \\
\text { monitoring }(\mathrm{h})\end{array}$ & $\begin{array}{l}\text { No. of } \\
\text { vectors }\end{array}$ \\
\hline Pt1 & 42 & M & C-4 & A & A & 9 & 49 & 141 \\
Pt2 & 19 & M & t-8 & A & A & 89 & 31 & 139 \\
Pt3 & 37 & M & t-1 & A & B & 86 & 24 & 29 \\
Pt4 & 49 & M & c-5 & A & A & 4 & 58 & 324 \\
Pt5 & 55 & M & t-1 & A & A & 3 & 81 & 104 \\
Pt6 & 25 & M & t-3 & A & A & 24 & 26 & 232 \\
Pt7 & 19 & M & C-5 & A & A & 51 & 31 & 233 \\
Pt8 & 40 & M & t-4 & A & A & 34 & 48 & 78 \\
Pt9 & 29 & M & t-12 & A & A & 46 & 74 & 317 \\
Pt10 & 45 & M & c-6 & A & C & 44 & 33 & 183 \\
Pt11 & 31 & M & t-4 & A & A & 22 & 15 & 223 \\
Pt12 & 21 & M & c-5 & B & B & 37 & 48 & 136 \\
Pt13 & 70 & F & C-4 & C & C & 2 & 30 & 128 \\
Pt14 & 51 & M & I-1 & A & C & 32 & 49 & 155 \\
Pt15 & 26 & M & t-5 & B & B & 22 & 38 & 72 \\
Pt16 & 47 & M & t-12 & C & C & 3 & 30 & \\
\hline & & & & & & & & 126 \\
\hline
\end{tabular}

AIS, American spinal cord injuries Impairment Scale; $c$, cervical; d, days; F, female; h, hours; l, lumbar; M, male; no, number; Pre-op, pre-operative; Pt., patient; $t$, thoracic; $w$, weeks; $y$, years. *From injury site

This article is protected by copyright. All rights reserved. 

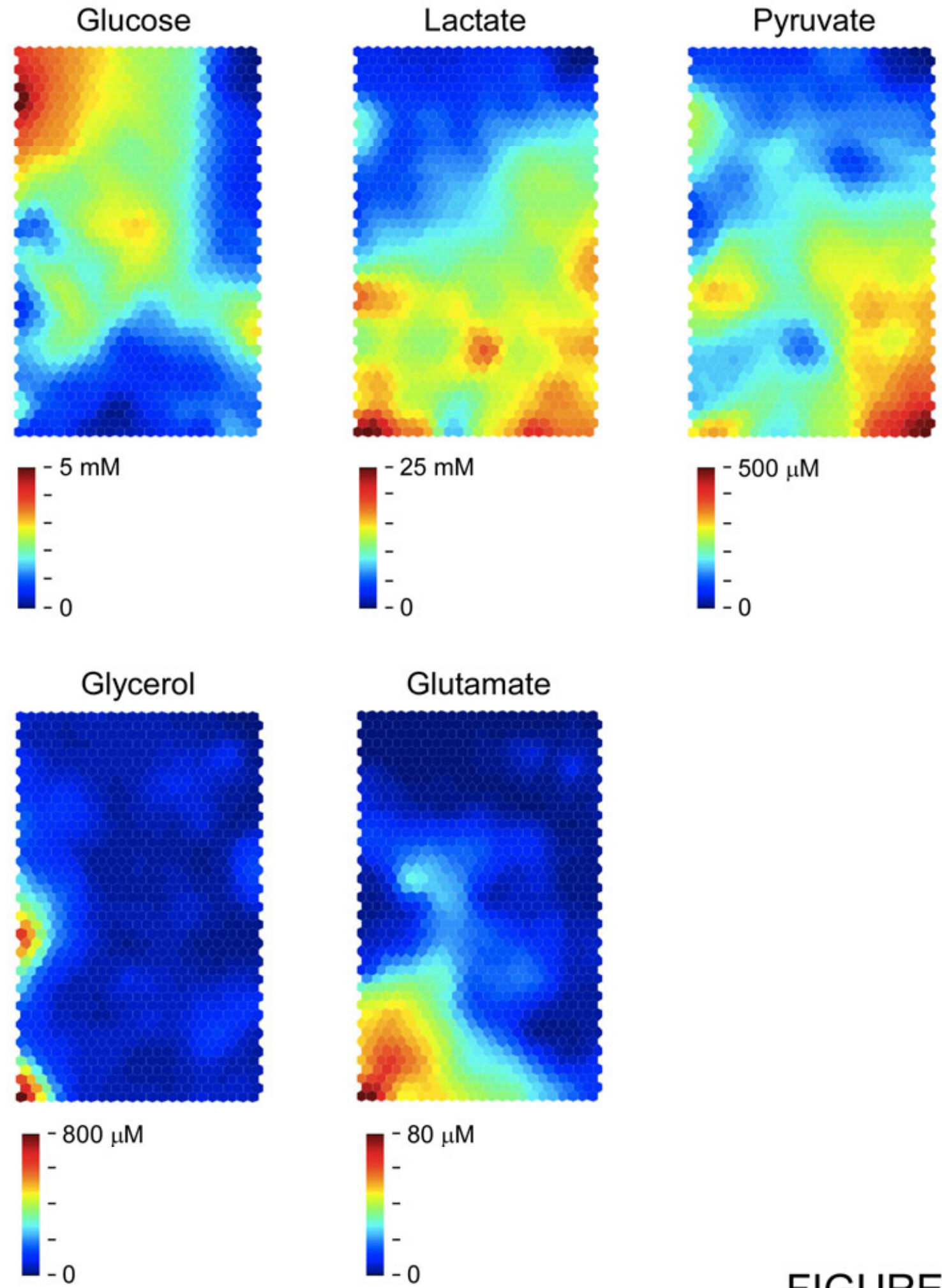

FIGURE 1

This article is protected by copyright. All rights reserved. 

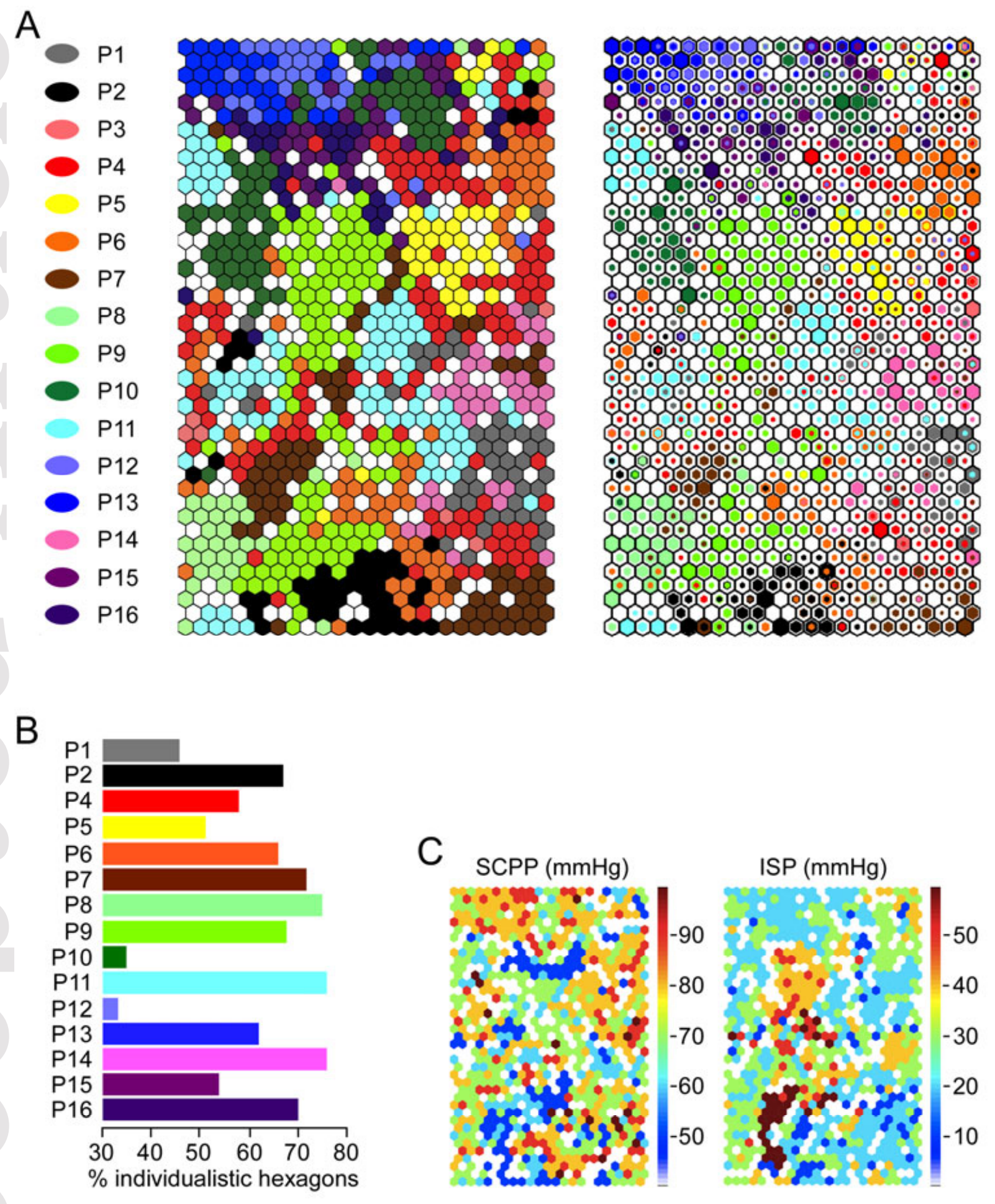

FIGURE 2

This article is protected by copyright. All rights reserved. 


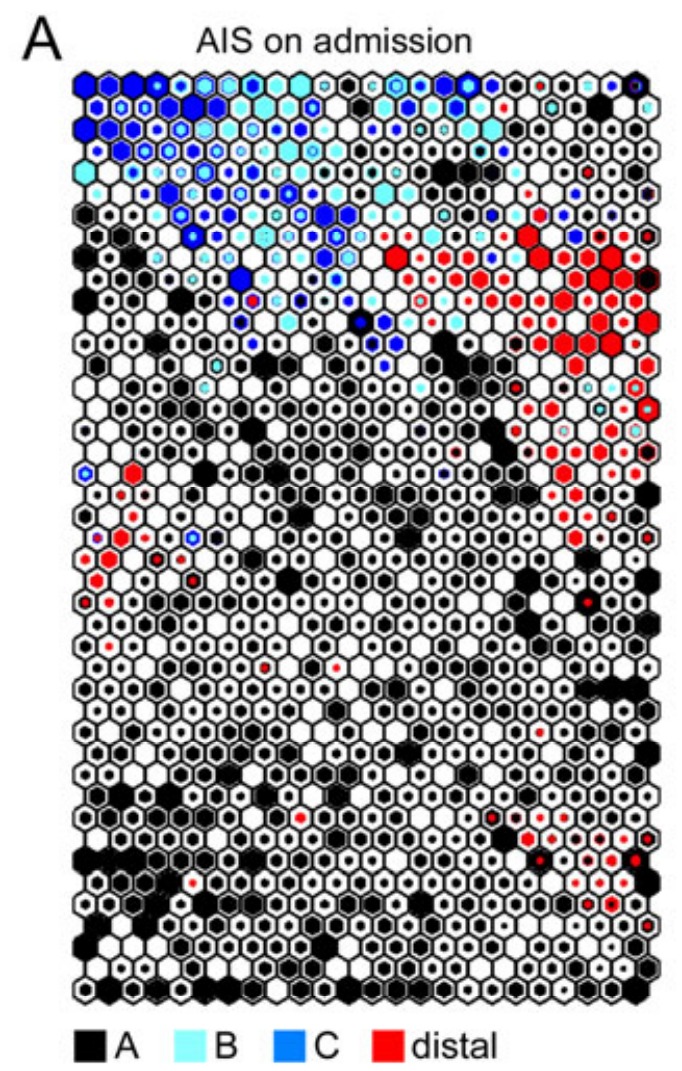

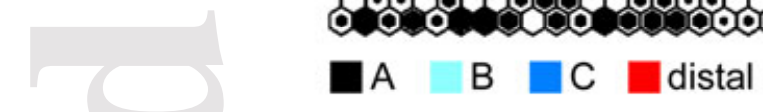

C

$\%$ hexagons per grid quadrant

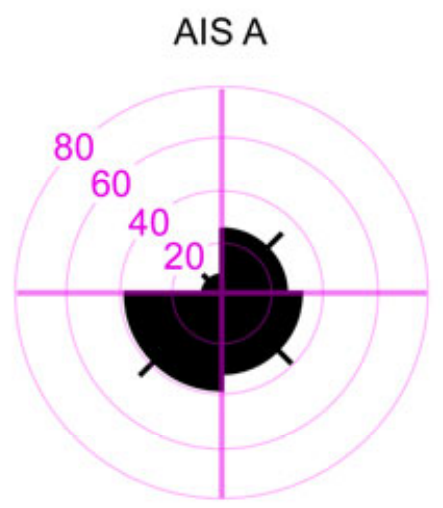

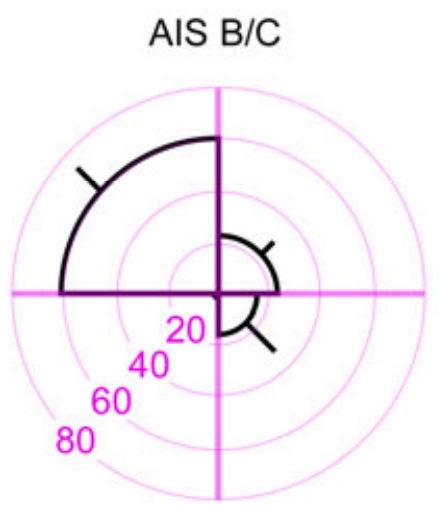

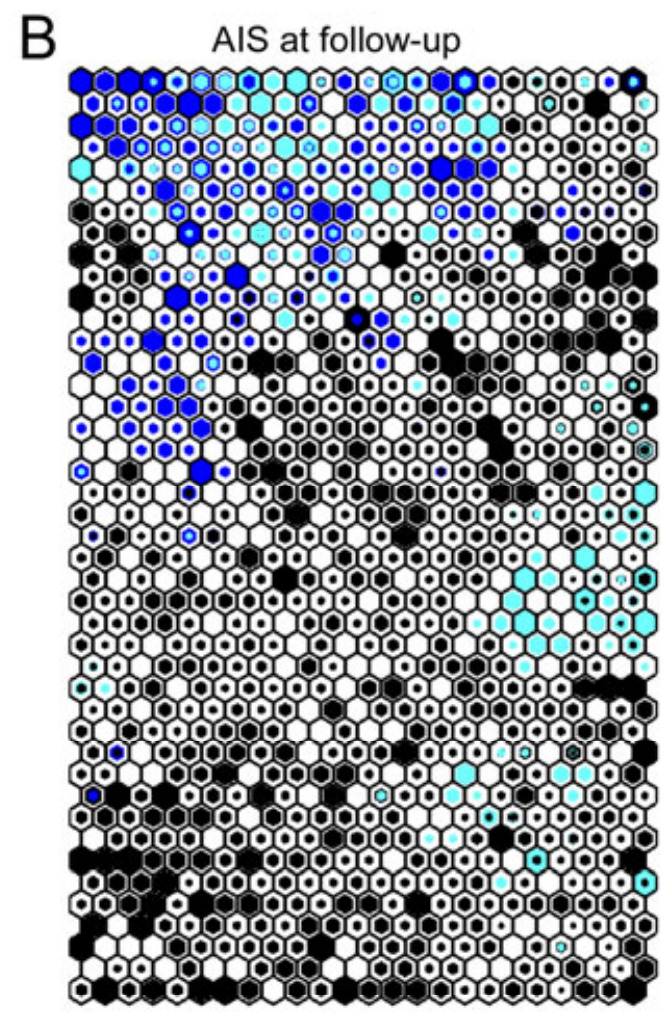

-A B C
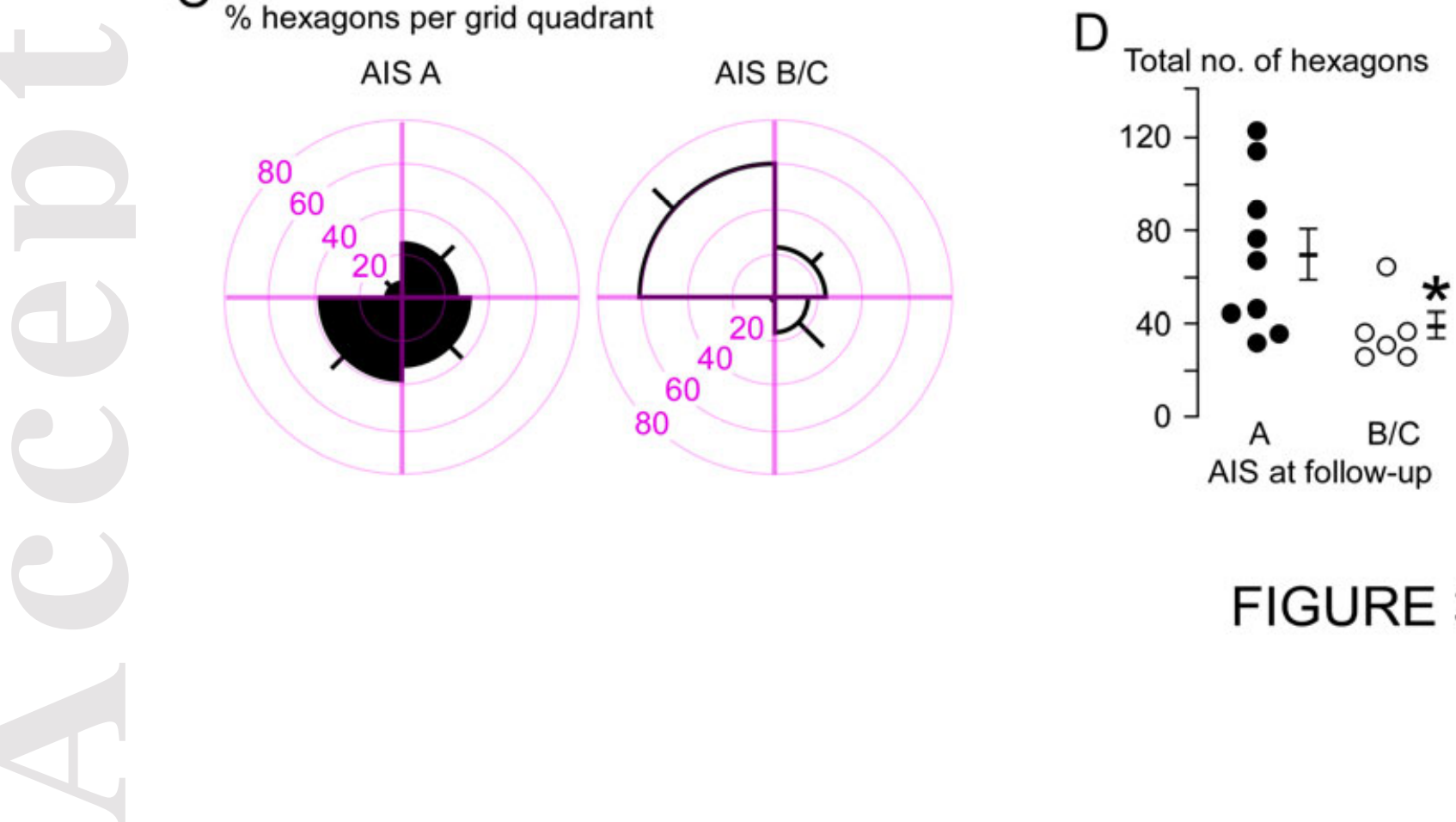

FIGURE 3

This article is protected by copyright. All rights reserved. 